\title{
AP0 Target Vault Shielding Study
}

F. M. Bieniosek

August 16, 1991

\section{Introduction and Summary}

Efforts are currently underway to redesign the neutron covers [Ref. 1] to meet radiation-safety guidelines, with the goal of reducing the radiation level over the vault by at least a factor of 100 . This note describes the results of measurements of radiation attenuation by sample shields. The purpose of the shielding study is to provide data for the redesign of the neutron covers.

The radiation escaping from the neutron covers appears to be predominantly gammas. In addition, there is a small, more penetrating component, which may consist of energetic $(>200 \mathrm{MeV})$ neutrons leaking past the neutron covers. Reduction of the measured radiation by more than an order of magnitude is easily achieved by placing a 6-inch steel plate over the existing neutron covers. However, the desired reduction by two orders of magnitude requires further attenuation of the more penetrating component. The conclusion of the study is that 48" of concrete should adequately attenuate the radiation.

\section{Shielding Calculations}

Reference 2 contains information on the radiation diffusing through the 6-ft steel shield modules over the target pit. Two conclusions may be drawn from that work. First, the neutron covers greatly reduce the neutron flux that impinges on them. Second, the ratio of gamma-ray flux to neutron flux above the neutron covers appears to be at least 10 .

\section{(a) Gammas}

Transmission of gamma rays through a shield of thickness $x$ may be calculated as [Refs. 3,4]

$$
\mathrm{T}(\mathrm{x})=\mathrm{B} \exp (-\mathbf{u} \mathrm{x})
$$

where $B$ is the build-up factor (a function of photon energy, shield thickness, material, and geometry), and $u$ is the gamma-ray absorption coefficient. The energy of gammas emitted in neutroncapture processes depends on the material; they typically range in 
energy up to about $2 \mathrm{MeV}$, but some materials (such as Fe) generate gammas up to $10 \mathrm{MeV}$. In the absence of a measurement of the gamma spectrum, we must be prepared to shield throughout the range $0.48-10 \mathrm{MeV}$.

(b) Neutrons

Some neutrons are leaking past the existing neutron cover, as shown in Ref. 2. Neutron scattering/attenuation calculations are more complicated than the equvalent gamma-ray calculations. Therefore, no detailed neutron transmission curves were calculated. However, design procedures discussed in the references are useful for determining the appropriate amount of shielding (see below). For example, concrete is a more effective neutron absorber, and therefore would be preferable over steel, because of the presence of hydrogen in the concrete.

\section{Experiment}

A study was performed to measure the transmission of ionizing radiation through several test shields. The test setup is shown in Fig. 1. Shields (18" or 36" concrete blocks; 6" to 10 " of steel plates in 2" increments) were placed on the upstream and downstream neutron covers, approximately over the beam path. An ionization chamber (chipmunk) was placed on the shield, and was in turn covered by the cover of a lead-lined (2.5" thick) coffin. The coffin was used to prevent scattered radiation from entering the radiation detector. Results from the tests are shown in Table 1. The unshielded dose rate data are in rough agreement with previous measurements (Ref. 5). The ratios of counts with shielding to counts without shielding are plotted as a function of shield thickness, in Fig. 2 for the case of steel, and in Fig. 3 for the case of concrete. Also plotted are the expected gamma-ray transmission curves, as calculated above. Measured transmission is in most cases higher than expected, and the addition of incremental shielding is fairly ineffective, in disagreement with the gamma-ray model. (The 36 " concrete test is a special case -- because of its square (36" wide) cross section, the block cannot be treated as a uniform shield.) The discrepancy is quantified in Fig. 4. There is agreement between model and data under the assumption that an additional component $(0.024$ of total upstream; 0.032 of total downstream), unaffected by the steel shields, is present.

\section{Interpretation of the Results}

Adding 18" (46 cm) of concrete under 8 " of steel reduces the flux of the unaccounted-for radiation by roughly $1 / 2$. It is interesting to note that this result is in agreement with published curves [Ref. 6] of shielding effectiveness for high-energy neutrons in ordinary 
concrete (Fig. 5), which show a half-value thickness of $48 \mathrm{~cm}$. Hence the penetrating radiation may consist of these energetic neutrons.

A general-purpose shield can be constructed of concrete. Neutrons of energy less than $1 \mathrm{MeV}$ should be eliminated in approximately the first $20 \mathrm{~cm}$. (The precise rate of attenuation depends on the moisture content of the concrete.) Gamma rays of energy $2 \mathrm{MeV}$ are eliminated in the first $70 \mathrm{~cm}$. Finally, the penetrating component is attenuated very slowly. The initial, unattenuated level is not well known. However, if we estimate the unshielded level to be in the range of 0.04 , it should be attenuated to less than 0.01 after $120 \mathrm{~cm}$ of concrete. Based on this prediction, a proposed shield, consisting of $48^{\prime \prime}(122 \mathrm{~cm})$ of concrete, is shown in Fig. 6 . Because of the attenuation of neutrons in the concrete, the new shield may replace the existing neutron covers.

\section{References}

1. P. Hurh, AP0 Target Hall Vault Shielding Upgrade Design Report, Accelerator Division Mechanical Support Department, Spec. \#1321-ES-296053, April 15, 1991.

2. P. Yurista and A. Elwyn, Radiation Physics Note \#73, June 2, 1988.

3. H. Goldstein, The Attenuation of Gamma Rays and Neutrons in Reactor Shields, US Atomic Energy Commission, May 1, 1957.

4. H. Etherington, ed., Nuclear Engineering Handbook, McGrawHill 1958.

5. M. Halling, Summary of PBAR Radiation Studies, memo 4/9/90

6. R. G. Jaeger, ed., Engineering Compendium on Radiation Shielding, Vol III, IAEA, Springer Verlag, NY 1968. 


\section{TABLE 1}

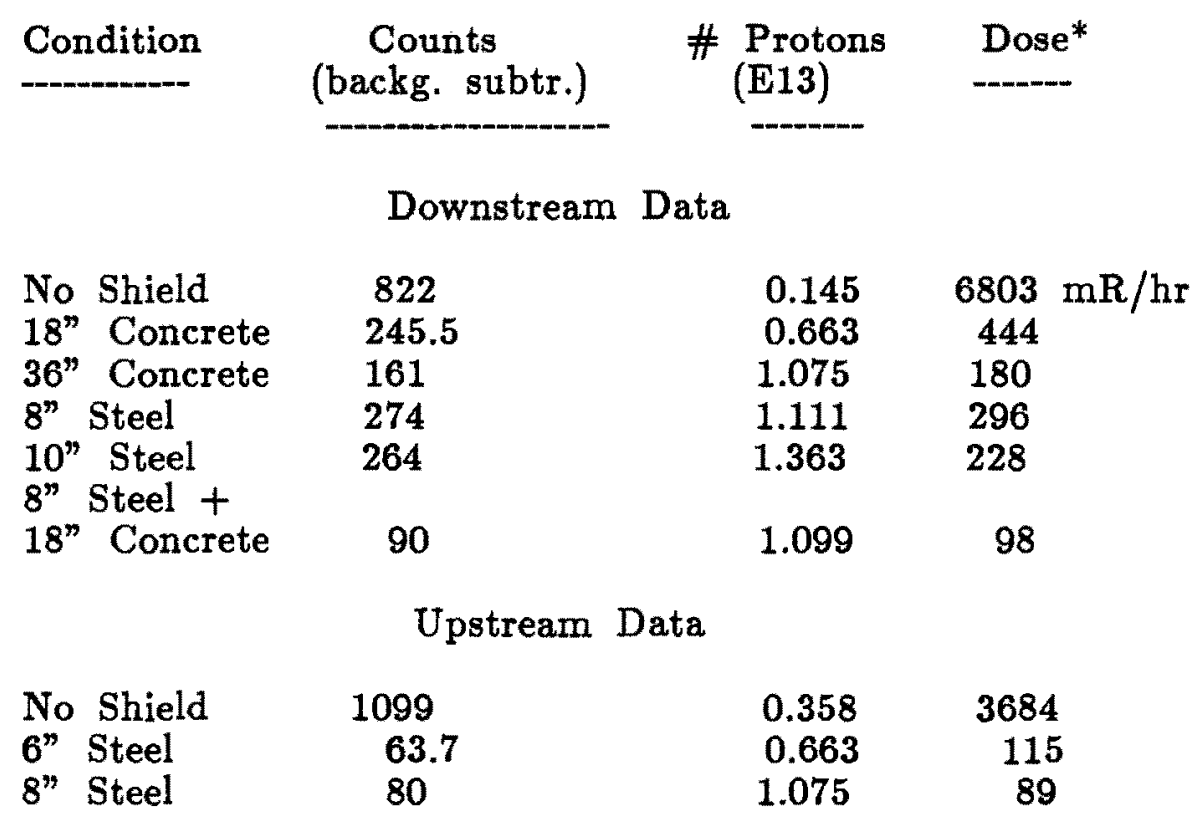

* $\overline{\text { Scaled }} \overline{\text { to }} \overline{3 \mathrm{E}} \overline{12}$ protons per pulse, 2 seconds per pulse. 


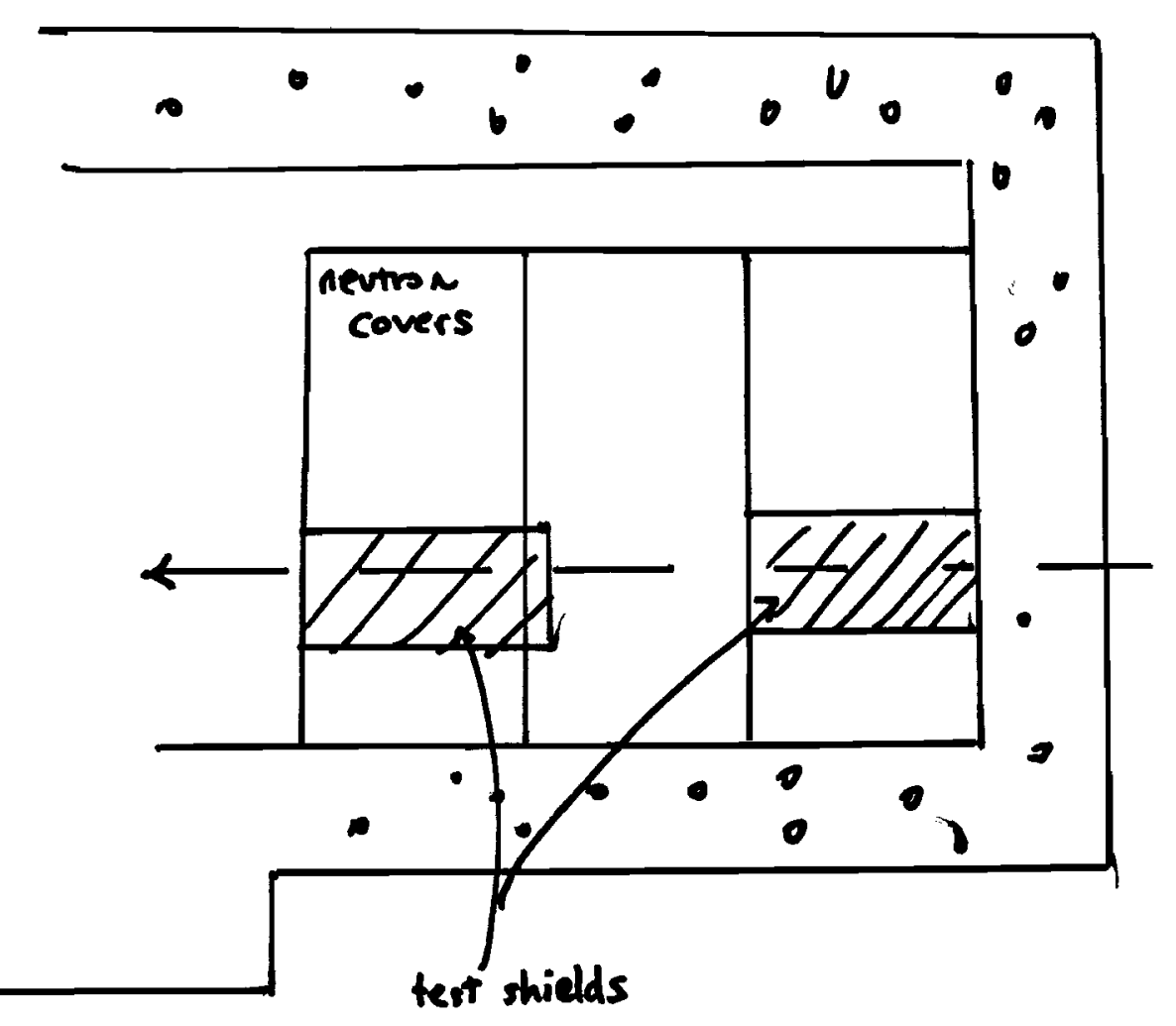

\section{Test Shield Pieces}

- $18^{\prime \prime} \times 36^{\prime \prime}$ Concrete Block

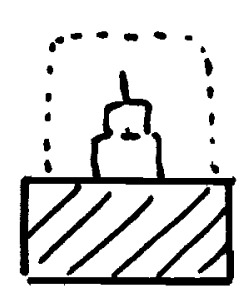

- $36^{\prime \prime} \times 36^{\circ}$ Coneroto Block

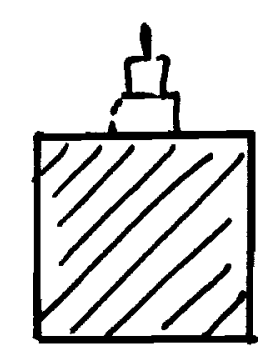

- (5) $2^{\prime \prime} \times 26^{\prime \prime}$ Steel Plates

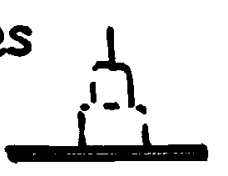




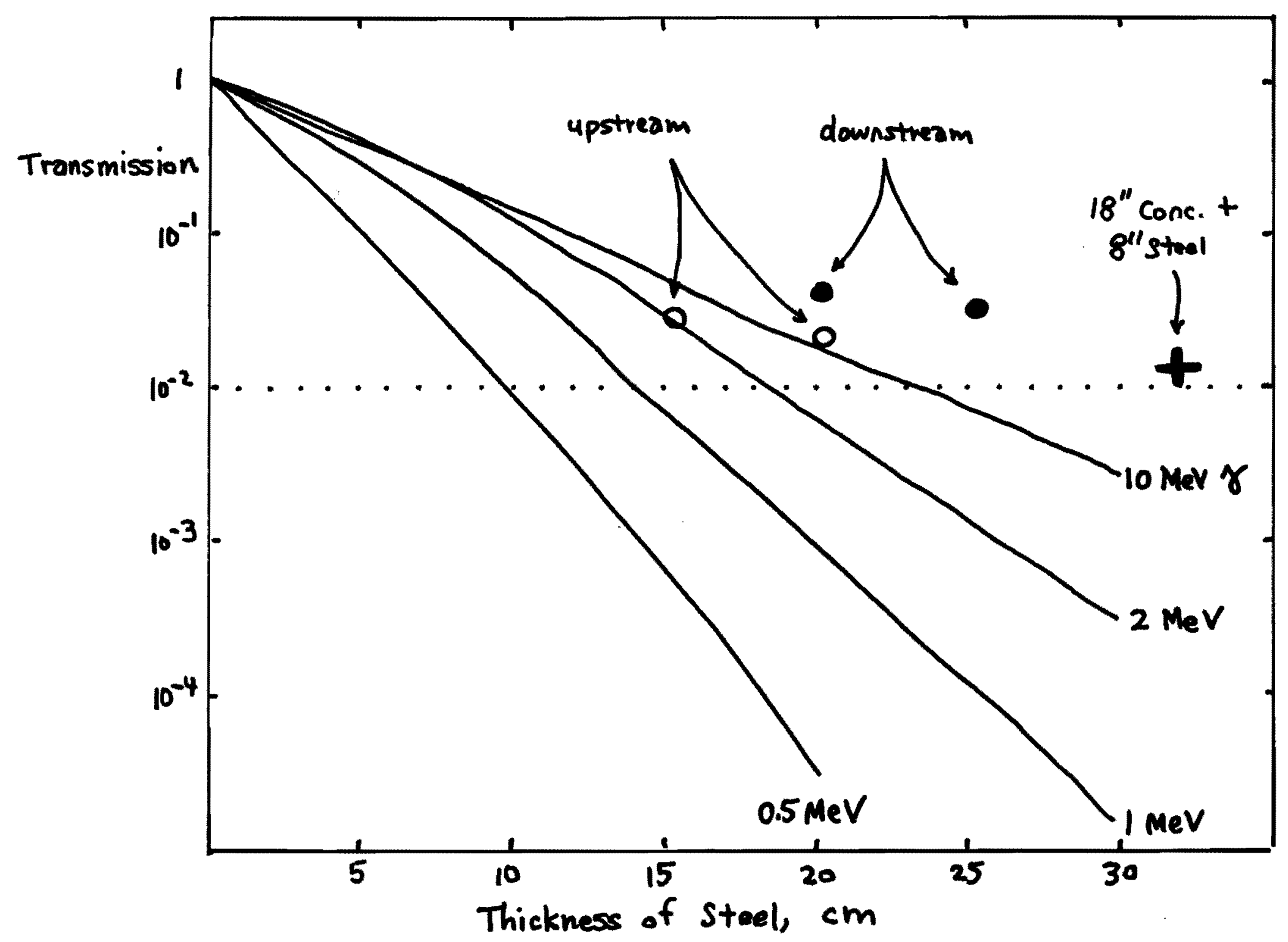

FIGURE 2. Comparison of data with gamma-ray transmission: steel. 


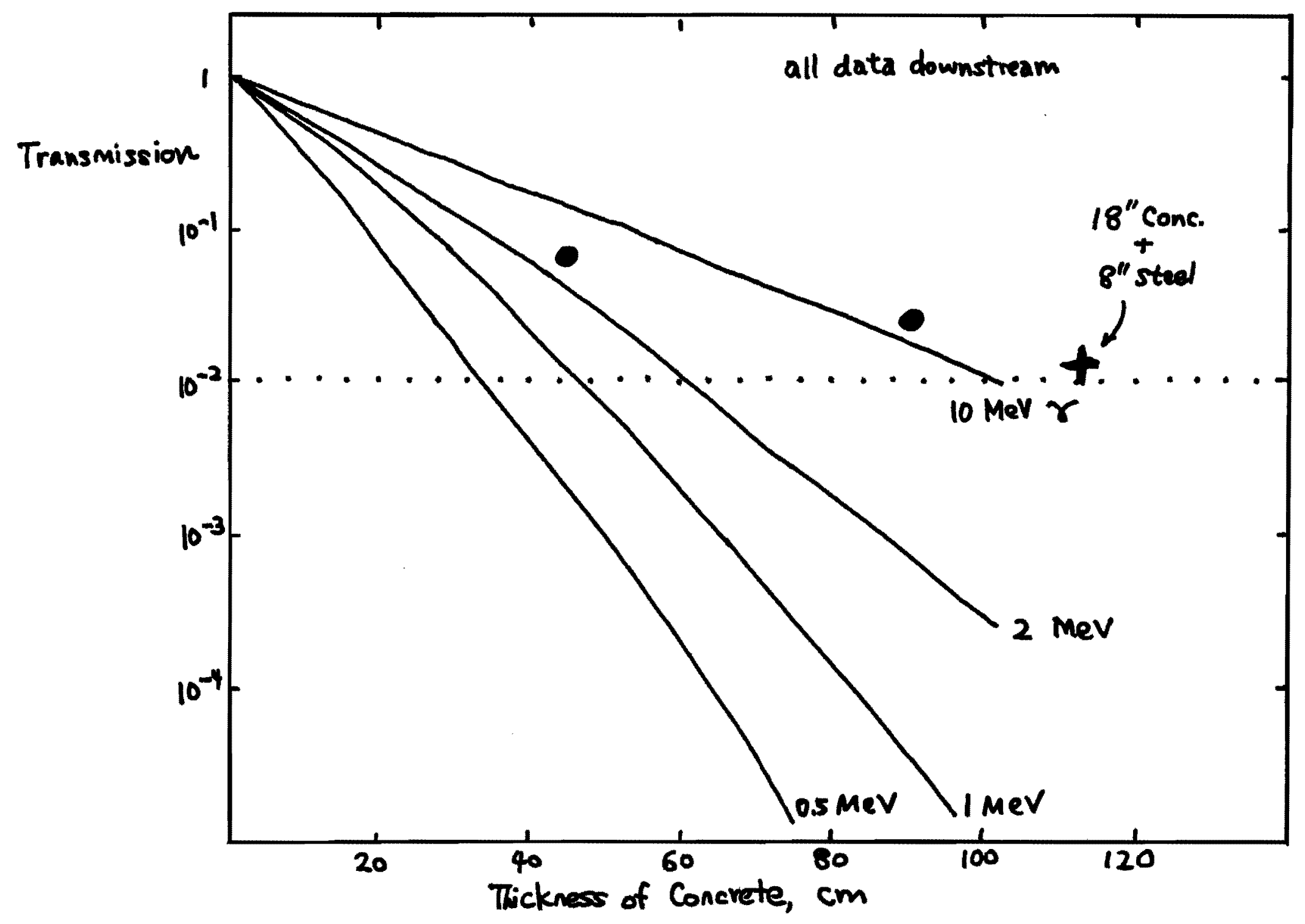

FIG. 3. Comparison of data with gamma-ray transmission: concrete 


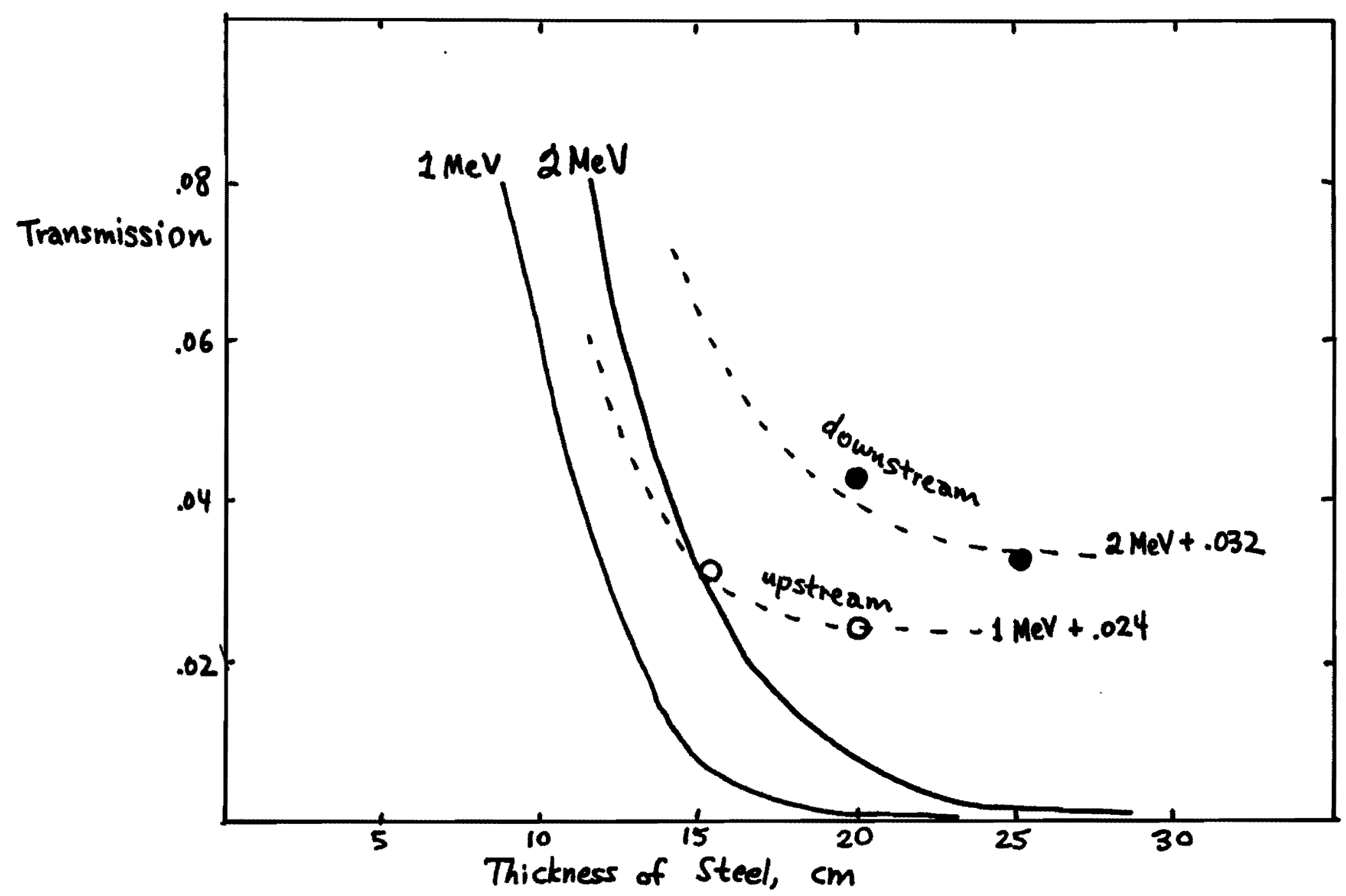

FIGURE 4. Discrepancy between data and gamma-ray transmission. 
because neutrons of lower energy have attenuation lengths substantially shorter than those with ener. gies above $150 \mathrm{MeV}$. It is only this penetrating high-energy component that determines the shield thickness, as can be seen in Figs. 10.7.-13 and -14 .

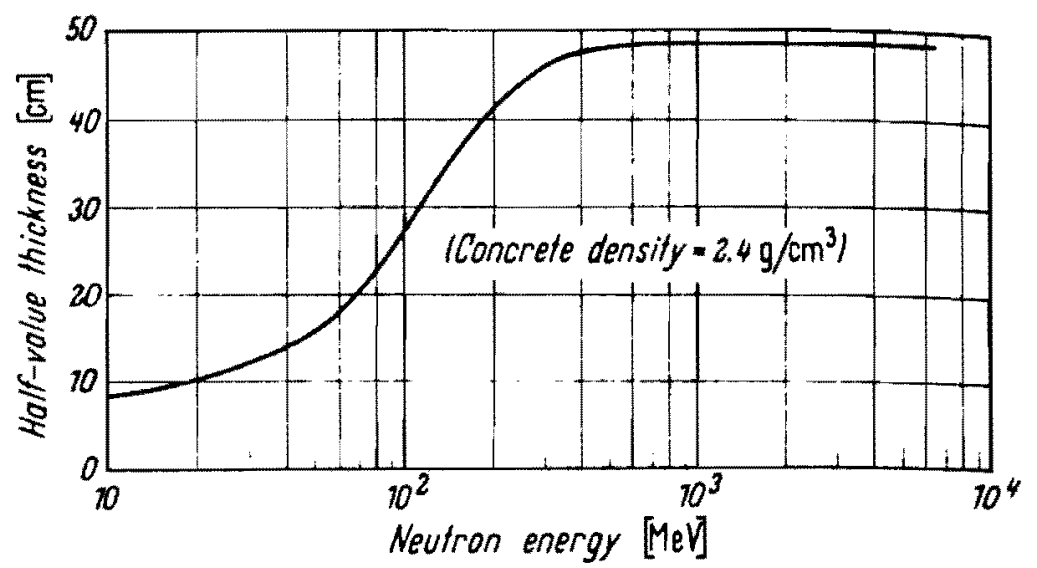

Fig. 10.7.-13. Half-value thickness for high-energy neutrons in ordinary concrete.

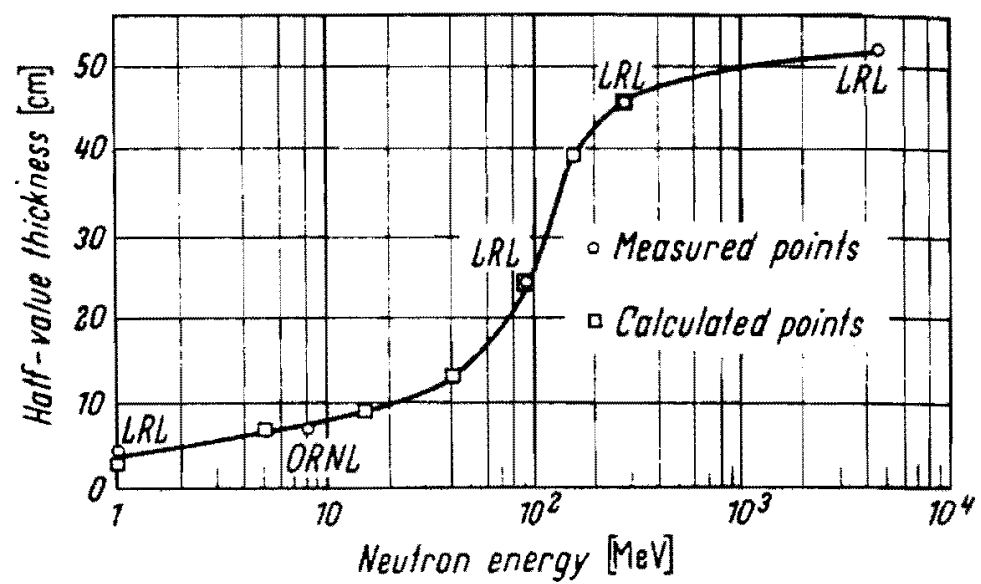

Fig. 10.7.-14. Attemuation of neutrons in ordinary concrete. At 90 and $270 \mathrm{MeV}$, measurements were made at the 184-inch $340-\mathrm{MeV}$ cyclotron. At $4.5 \mathrm{GeV}^{\prime}$ the measurement was made at the Bevatron.

Engineering Compendium on Radiation Shielding, Vol III R.G. Joejer, ed. 1908 p. 158.

FIGURE 5. Attenuation of neutrons in ordinary concrete. 


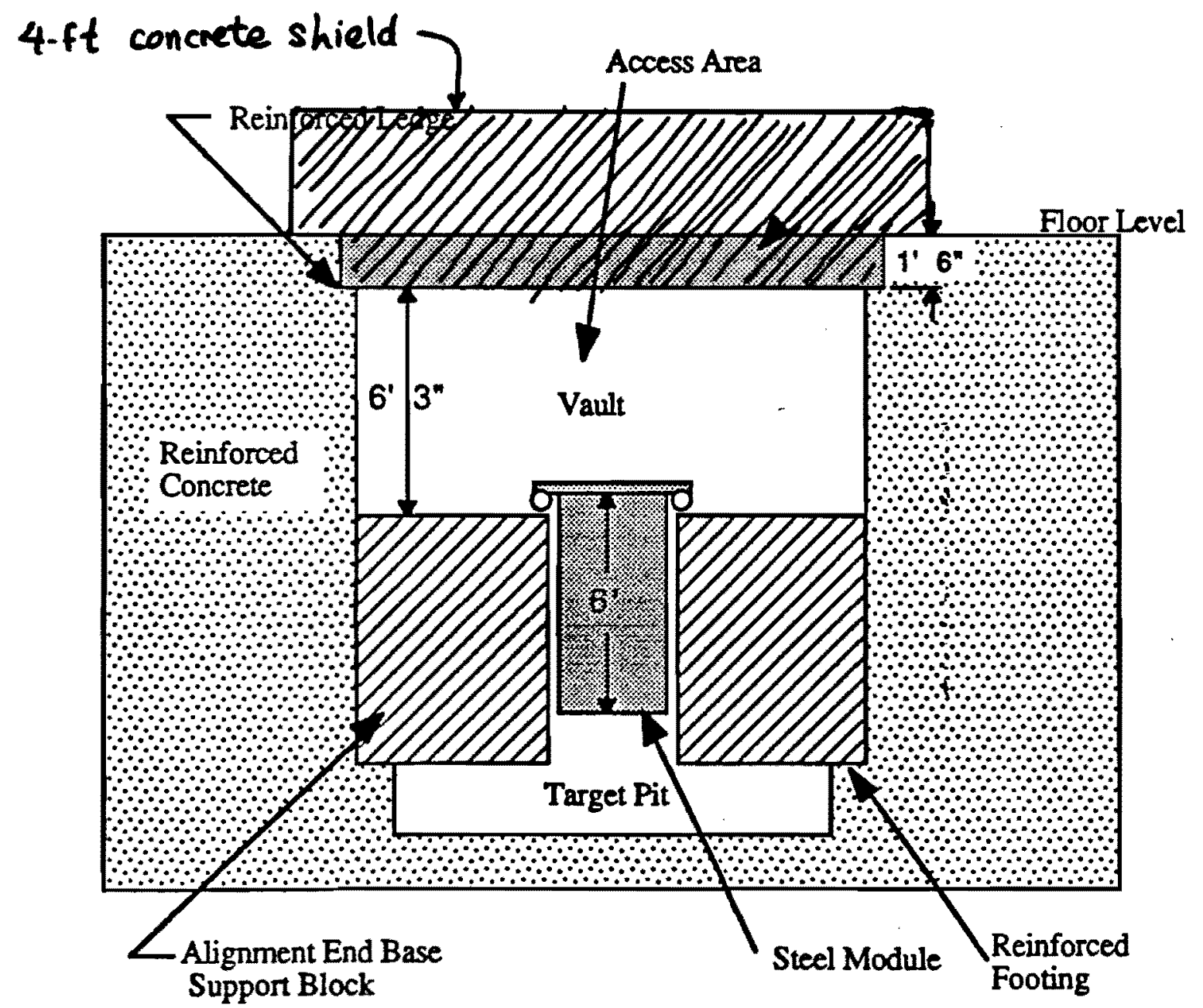

FIGURE 6. Elevation schematic of vault showing proposed 4-ft. concrete shield. 
Test Shield Pieces

- $18^{\prime \prime} \times 36^{\prime \prime}$ Concrete Block

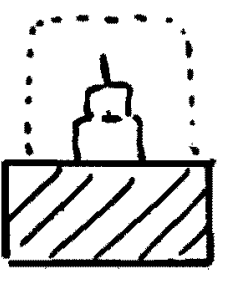

- $36^{\prime \prime} \times 36^{\prime \prime}$ Concreto Block

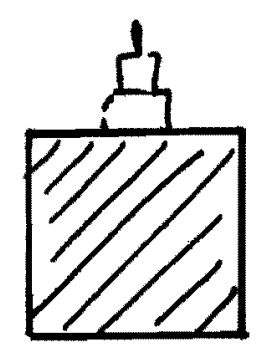

- (5) $2^{\prime \prime} \times 26^{\prime \prime}$ Steel Plates

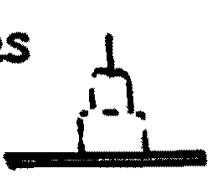




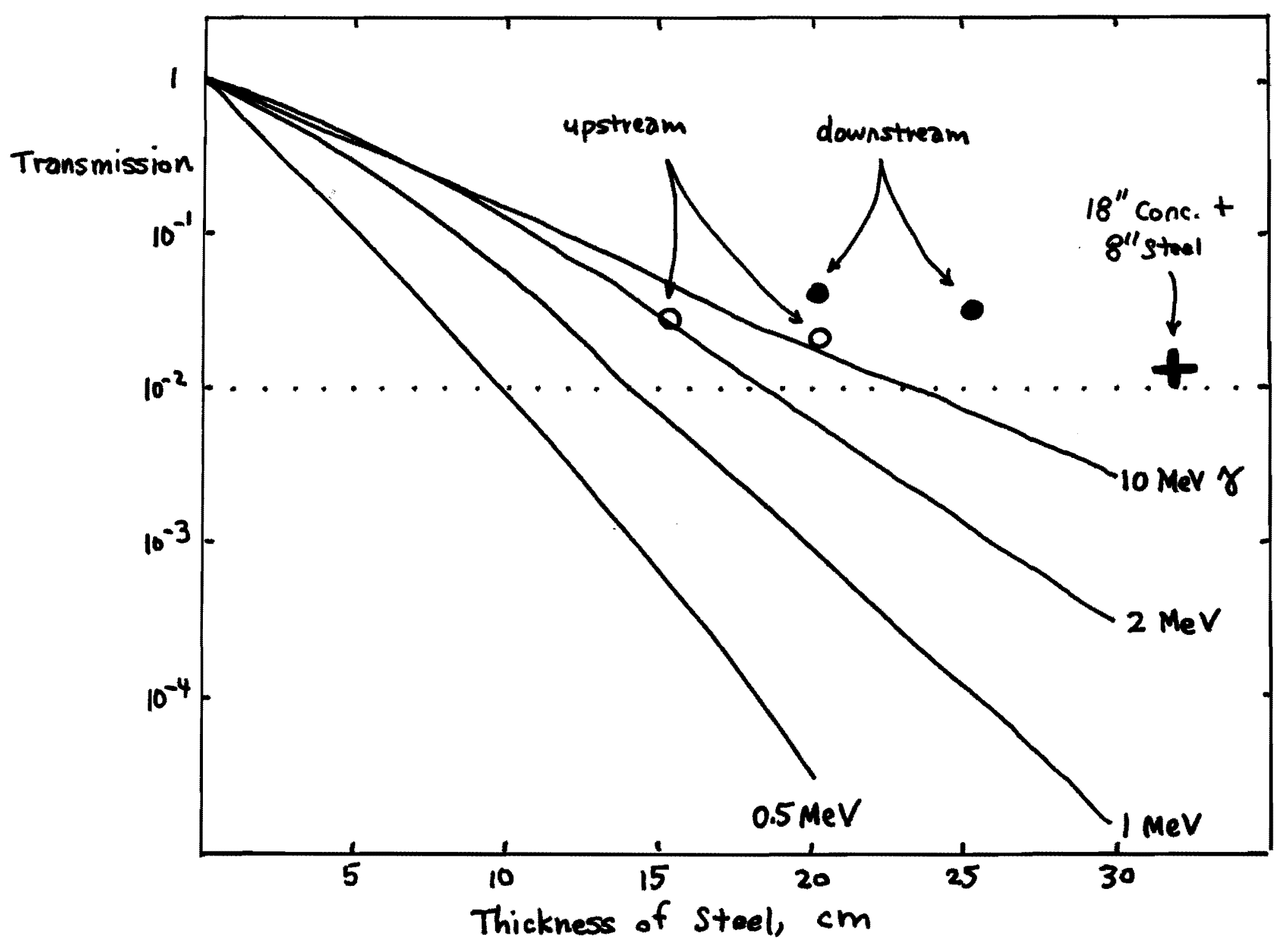

FIGURE 2. Comparison of data with gamma-ray transmission: steel. 


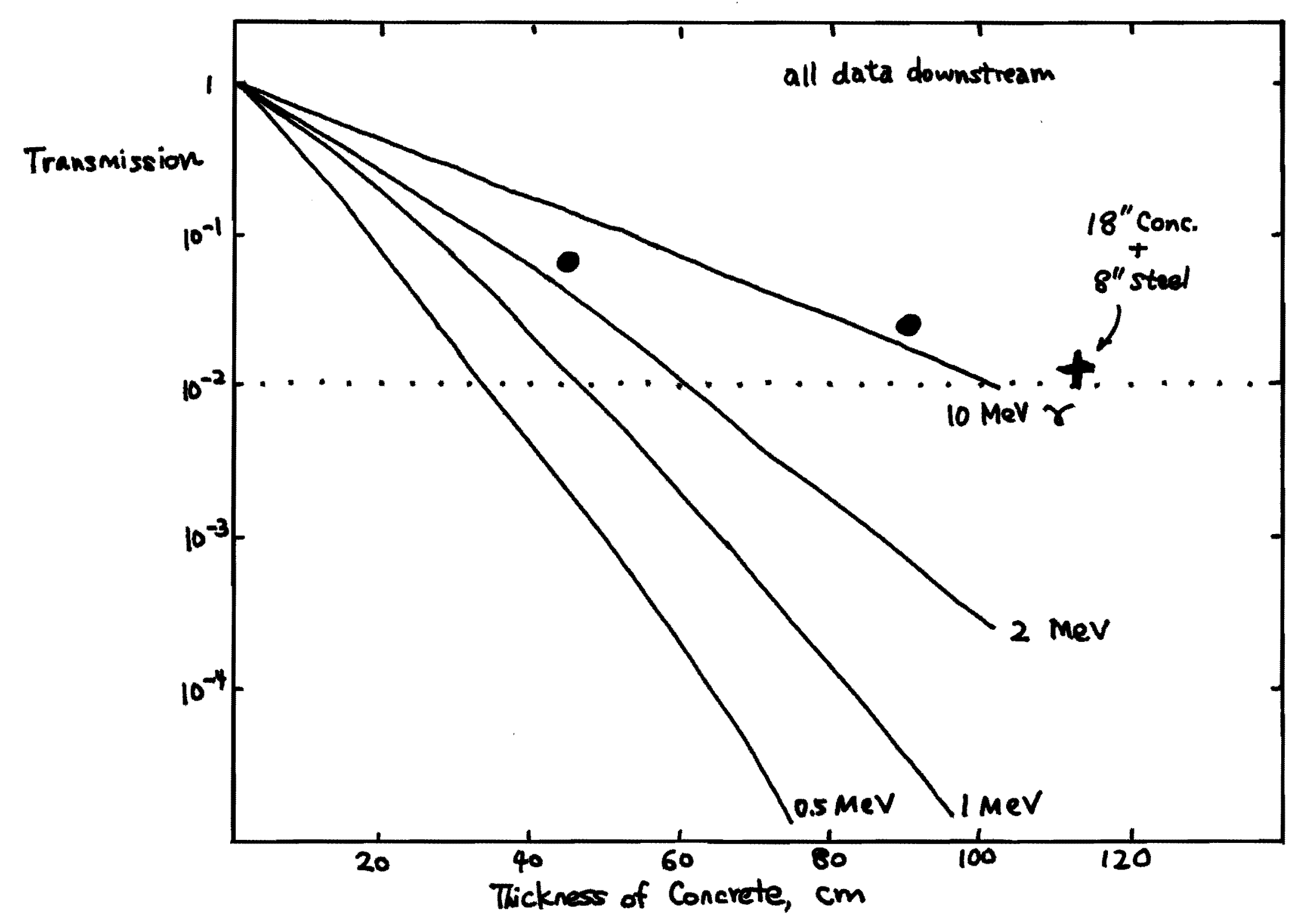

FIG. 3. Comparison of data with gamma-ray transmission: concrete 


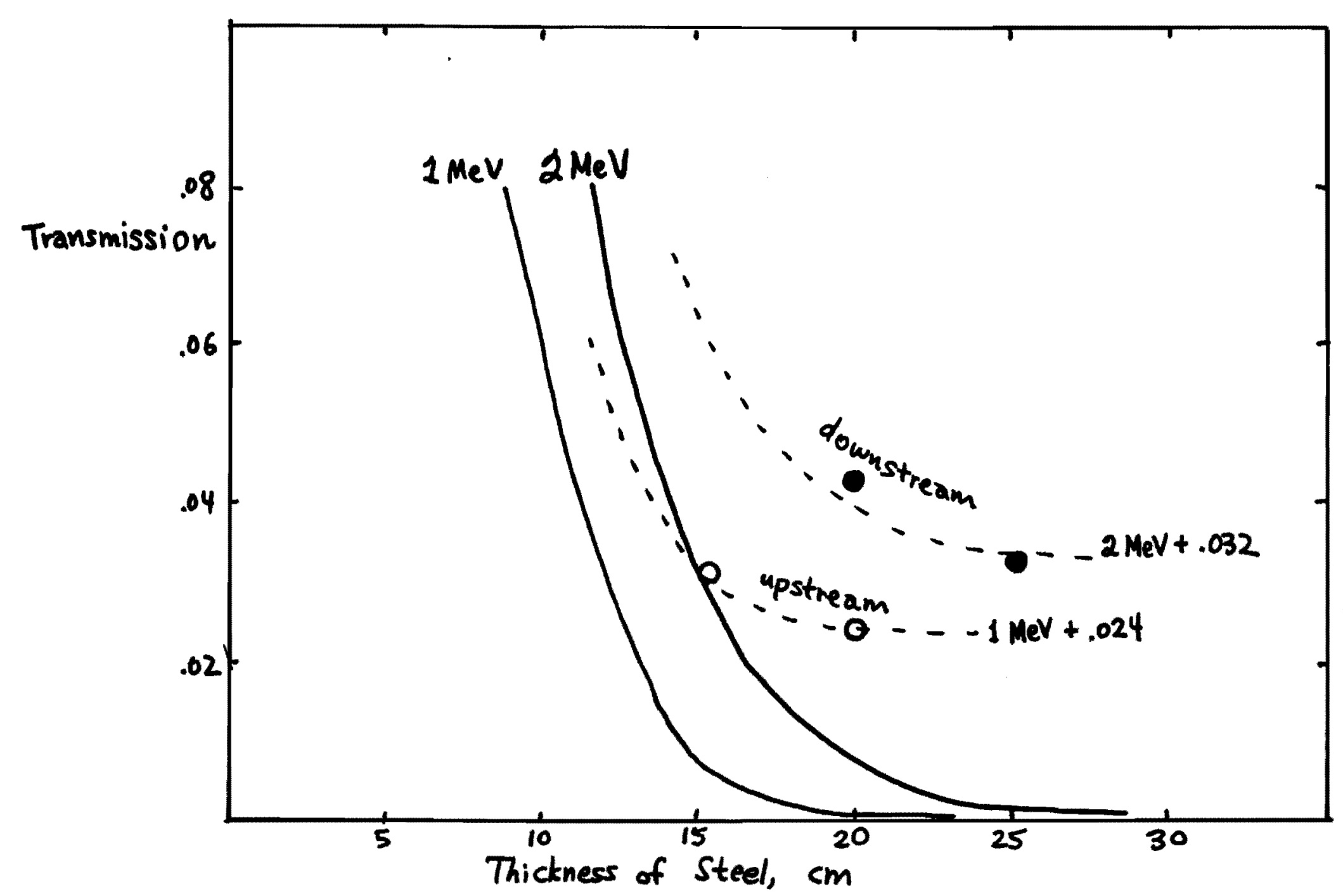

FIGURE 4. Discrepancy between data and gamma-ray transmission. 
because neutrons of lower energy have attenuation lengths substantially shorter than those with energies above $150 \mathrm{MeV}$. It is only this penetrating high-energy component that determines the shield thickness, as can be seen in Figs. 10.7.-13 and -14 .

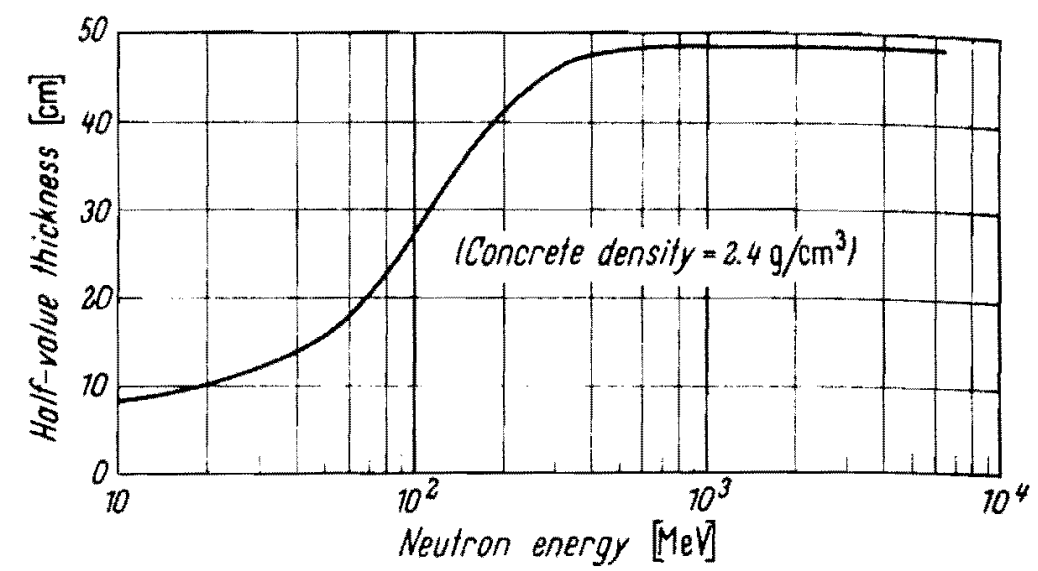

Jig. 10.7.-13. Half-value thickness for high-energy neutrons in ordinary concrete.

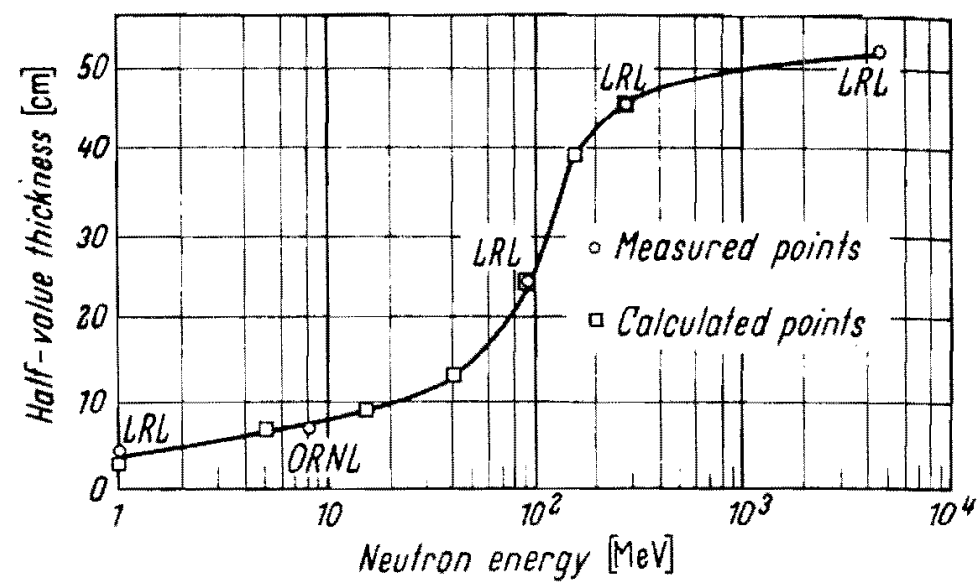

Fig. 10.7.-14, Attenuation of neutrons in ordinary concrete. At 90 and $270 \mathrm{MeV}$, measurements were made at the 184 -inch $340-\mathrm{MeV}$ cyclotron. At $4.5 \mathrm{GeV}$ the measurement was made at the Bevatron.

Engineering Compendium on Radiation Shietting, Vol III R.G. Jaeger, ed., 1908 p. 158.

FIGURE 5. Attenuation of neutrons in ordinary concrete. 


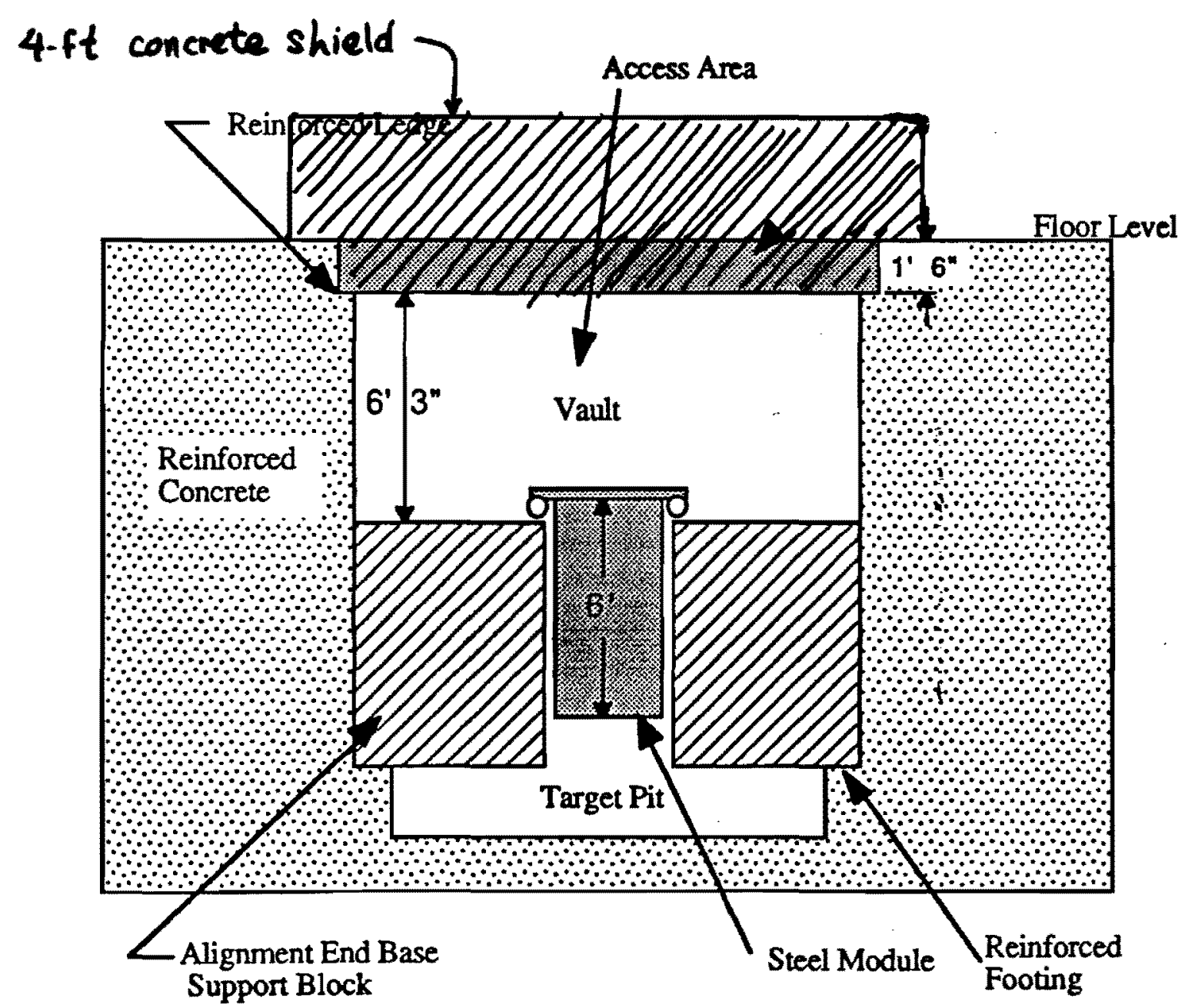

FIGURE 6. Elevation schematic of vault showing proposed 4-ft. concrete shield. 